\title{
Screening of silent myocardial ischaemia in type 2 diabetic patients with additional atherogenic risk factors: applicability and accuracy of the exercise stress test
}

\author{
A Doyle, C Kelly, A Bridges and S B M Reith \\ Diabetes Centre, Stirling Royal Infirmary, Livilands, Stirling FK8 2AU, UK \\ (Correspondence should be addressed to A Doyle, Division of Cardiovascular Sciences, Gardner Institute, Western Infirmary, Glasgow G11 6NT, UK; \\ Email: arthurspdoyle@hotmail.com)
}

The recent article by Bacci et al. in European Journal of Endocrinology (1) on the use of exercise ECG in type 2 diabetes raises some interesting points for discussion. The authors report the value of a positive result from exercise ECG in predicting the presence of significant coronary artery disease (CAD) at angiography. The authors report their results of a cross-sectional study of patients with type 2 diabetes and additional risk factors for CAD undergoing exercise ECG. They undertook coronary angiography in those with a positive result and a selection of those with negative results. They express this as an 'accuracy' of $79 \%$ and a sensitivity of $71 \%$ for exercise ECG predicting significant CAD. This is far better than our experience and previous reports of this technique $(2,3)$.

The reason for the unusually high sensitivity lies in flawed interpretation of results. Not every subject underwent the gold standard of coronary angiography; only those with positive results and a selection of those with negative results. Therefore at best, only an estimate of the true sensitivity can be made. The authors estimate sensitivity only with reference to those patients who were selected for coronary angiography. Furthermore the authors do not take into account the failed and uninterpretable exercise ECGs or even the extent of false negative results influencing the sensitivity statistic. The results from this method are invalid. Table 1 shows our attempted reinterpretation of the results.

It can now be seen that exercise ECG fails to detect two-thirds of those with significant CAD. This is in keeping with our experience and other literature. These results along with our own experience suggest that the exercise ECG is a useful additional risk factor in selecting patients at significant risk from CAD, but not in isolation. As the authors mention, there is little reassurance from a negative exercise ECG. This is reinforced by other reports of the prognosis of diabetic patients with negative coronary screening by stress echocardiography (4).

In our population of 200 consecutive type 2 diabetics, $30 \%$ were positive yet $50 \%$ were inconclusive. Of those with positive results put forward for angiography $96 \%$ had $C A D$ requiring intervention. This group represented
Table 1 Reinterpretation of the results.

\section{$n=206$}

Positive tests = 27 (13.1\%); angiogram positive in 20/27 (74\%) Negative tests $=114(55.3 \%)$; angiogram positive in $8 / 44(18 \%)$

Failed/non-diagnostic tests $=65(31.6 \%)$

Number with angiographic disease in the positive group $=20$

Estimated total number with angiographic disease in the negative group $=20.5(18 \%)$

Estimated number with angiographic disease in the failed group $=18.6^{*}$

Estimated total prevalence of CAD in the study population $59.1 / 206=28.7 \%$

Therefore sensitivity $\left(20_{\text {(true positive) }}\right.$

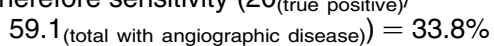

* Assuming the failed group is similar to the pooled positive and negative group, i.e. $28.7 \%$.

$13 \%$ of the population. We had no angiographic studies in the negative or non-diagnostic groups. We found a strong association between the number of additional risk factors and the result from exercise ECG. This was stronger even than the presence or absence of chest discomfort on detailed questioning.

To guide clinical practice, what is required is a prospective angiographic analysis of the type 2 diabetes patient population in relation to clinical risk factors and additional clinical tests. This would validate the clinical risk score proposed from United Kingdom Prospective Diabetes Study 56 (UKPDS 56) (5) that may more accurately select patients with CAD. Certainly, a positive exercise ECG suggests the need for angiography, although one could even justify simply considering angiography in all those with type 2 diabetes and two additional risk factors given that at least a quarter may have significant disease.

\section{References}

1 Bacci S, Villella M, Villella A, Langialonga T, Grilli M, Rause A et al. Screening of silent myocardial ischaemia in type 2 diabetic patients with additional atherogenic risk factors: applicability and accuracy 
of the exercise stress test. European Journal of Endocrinology 2002 147 649-654.

2 Doyle A, Kelly C, Bridges A \& Reith S. Unpublished data.

3 Weiner DA. Significance of exercise testing in patients with diabetes mellitus: a report from the Coronary Artery Surgery Study (CASS) Registry. American Journal of Cardiology 199168 729-734.

4 Kamalesh M, Matorin R \& Sawada S. Prognostic value of a negative stress echocardiographic study in diabetic patients. American Heart Journal $2002143163-168$.
5 Stevens RJ, Kothari V, Adler AI, Stratton IM \& Holman RR. (UKPDS Group), The UKPDS Risk Engine: a model for the risk of coronary heart disease in type II diabetes (UKPDS 56). Clinical Science $2001101671-679$.

Received 5 May 2003

Accepted 6 May 2003 